\title{
Asymmetric fiber taper for temperature sensing applications
}

\begin{abstract}
Optical fibers can be tapered in order to change their light coupling or light propagation properties. The evanescent wave can interact with environmental perturbations surrounding the waist region thus affecting the light propagation. This allows the waist region to be employed as an effective sensing region. This work is focused on a new fiber taper structure referred to as asymmetric fiber taper for temperature sensing application. Asymmetric tapered fiber is fabricated with different down-taper and up-taper lengths. By subjecting the sensor to temperature variation, wavelength shifts corresponding to different temperature can be observed. Comparison with symmetric taper sensor, asymmetric exhibits better sensitivity making it a feasible choice for this application. The best sensitivity is obtained from taper profile $2-15-8$ which is $0.2395 \mathrm{~nm} /{ }^{\circ} \mathrm{C}$.
\end{abstract}

Keyword: Asymmetric; Taped fiber; Fabrication 